\title{
Pericardial effusion and tamponade in a young woman
}

A young woman presented to the Singapore General Hospital with a history of cough in the 2 weeks prior. She had no significant past medical history and no history of immunocompromised state. Chest radiograph showed cardiomegaly. Electrocardiogram showed low voltages in precordial leads (Fig. 1A). A computed tomography scan of the chest was performed, which showed a low-density large pericardial effusion with enhancement of the thickened pericardium, as well as patchy consolidation in the lung bases with partial left lower lobe collapse (Fig. 1A).

She was initially covered with intravenous antibiotics (co-amoxiclav) and a pericardiocentesis was unsuccessfully attempted. The patient subsequently underwent an emergency left anterior thoracotomy, with the creation of a pericardial-pleural window. Intraoperatively, $650 \mathrm{~mL}$ of haemoserous pericardial effusion was drained. The pericardium was noted to be thickened with intrapericardial fibrin, and the epicardial surface of the heart was inflamed. The 2 specimens were sent to the anatomical pathology and microbiology laboratories, respectively. Gram stain of the pericardial fluid, however, was negative. After 72 hours of incubation, there was no growth of organisms from culture of the pericardial fluid, pericardial tissue or tissue from the intrapericardial fibrin, and the initial plates were discarded.

Histopathology review of tissue from the pericardial biopsy demonstrated fibrinous exudates on the visceral aspect, overlying granulation tissue with superimposed acute and chronic inflammation, and abscess formation. Within one of the abscess areas, a single branching filamentous bacterial colony was identified in the haematoxylin and eosin section, and positively highlighted by Grocott's methenamine silver (Fig. 1B). This finding was communicated to the microbiologist by the infectious disease physician. Pericardial tissue specimens were retrieved and re-inoculated on plates for prolonged incubation under conditions optimised for the recovery of anaerobic organisms (blood agar and MacConkey agar incubated at $35^{\circ} \mathrm{C}$ in $5 \% \mathrm{CO}_{2}$, and $\mathrm{CDC}$ anaerobe blood agar incubated anaerobically at $35^{\circ} \mathrm{C}$ ). On day 6 of re-incubation, 3 whitish colonies grew on the $\mathrm{CDC}$ anaerobe blood agar. Gram stain microscopy of the bacterial colony showed gram-positive bacilli (Fig. 1C).
What is your diagnosis?
A. Tuberculous pericarditis
B. Actinomycosis
C. Nocardiosis
D. Acute primary pneumococcal purulent pericarditis
E. Idiopathic purulent pericarditis; finding of isolated bacterial colony may be an incidental contaminant

Diagnosis: Anaerobic pericarditis caused by Actinomyces spp. presenting as cardiac tamponade. The finding of a single bacterial colony demonstrating filamentous architecture was consistent with a single sulphur granule typically formed by Actinomyces spp.

While tuberculous pericarditis could also sometimes present as gram-positive bacilli, smears for acid-fastbacilli and polymerase chain reaction (PCR) testing for Mycobacterium tuberculosis performed on pericardial fluid and tissue showed negative results.

While filamentous branching architecture can also be seen in cases of nocardiosis, nocardiosis typically manifests in immunocompromised hosts. Prolonged re-incubation of pericardial tissue specimens under anaerobic conditions yielded colonies of a gram-positive bacilli, which were identified as Actinomyces meyeri on matrix-assisted laser desorption/ionisation-time of flight (MALDI-TOF) mass-spectrometry analysis utilising the Bruker Biotyper system (Bruker Daltonics, Germany).

While pneumococcal infection is a common cause of purulent pericarditis, this would present as gram-positive cocci instead of gram-positive bacilli. Additionally, in-house partial $16 S$ ribosomal $R N A$ gene sequencing performed on pericardial tissue identified Actinomyces spp. and Fusobacterium spp. Periodontitis was noted on dental review. The patient received 6 weeks of intravenous ceftriaxone, followed by high-dose amoxicillin (1g 3 times a day) for another 6 months, with clinical and radiological resolution (Fig. 2). At 10-month follow-up, the patient remained well without relapse.

The presence of a rare, though compatible clinical syndrome (anaerobic pericarditis caused by Actinomyces spp. presenting as cardiac tamponade), and concordance 

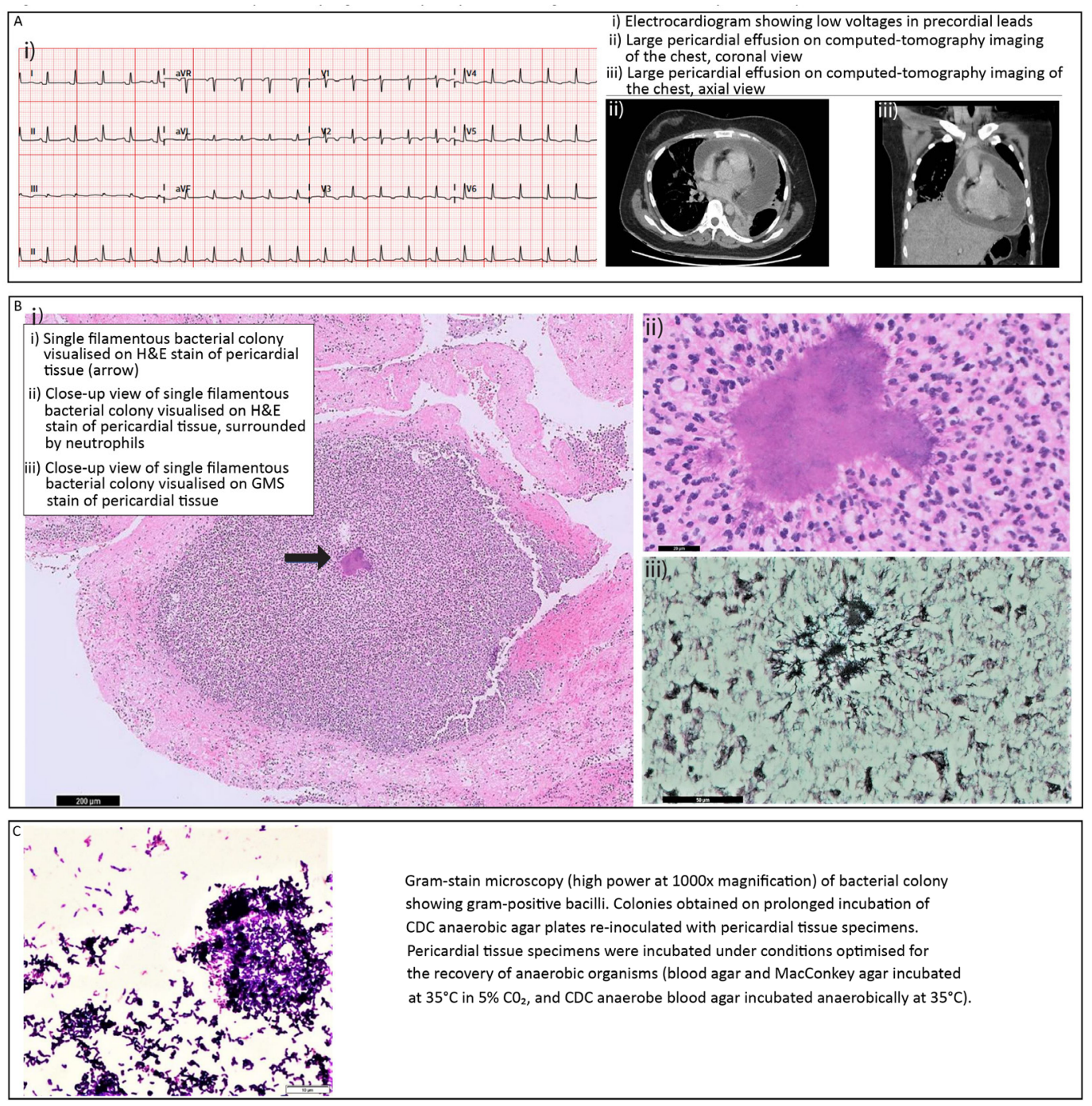

Fig. 1. Pericardial effusion and cardiac tamponade in a young immunocompetent patient with a single filamentous bacterial colony identified on pericardial tissue.

GMS: Grocott's methenamine silver; H\&E: haematoxylin and eosin
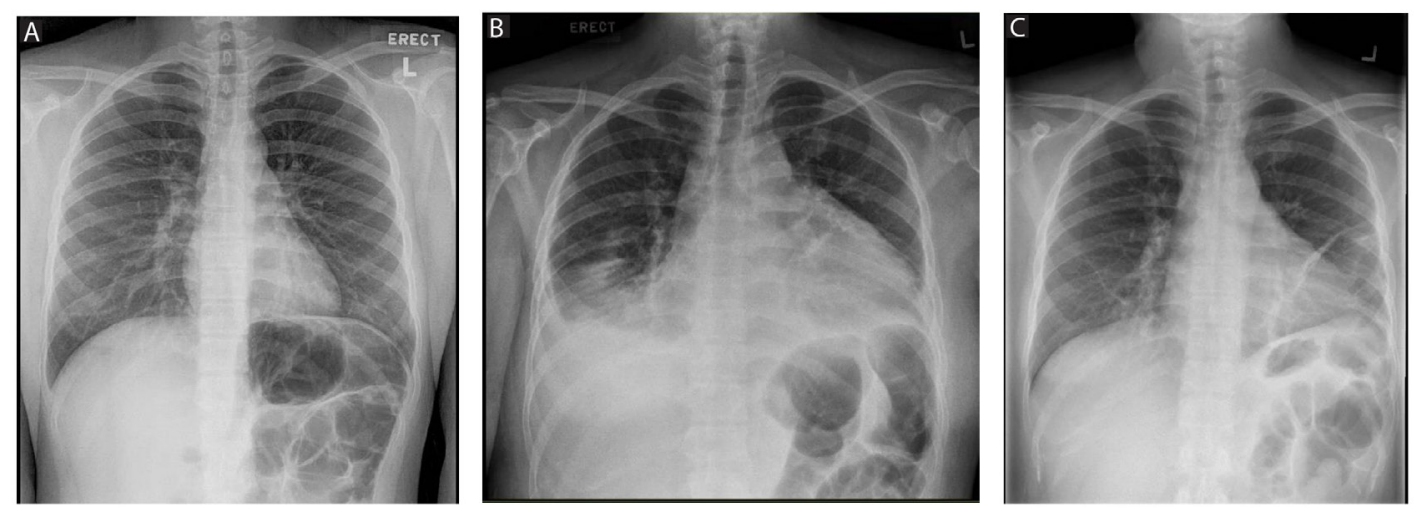

Fig. 2. Radiological progression and resolution of pericardial effusion in a young immunocompetent patient caused by Actinomyces meyeri. (A) Chest radiograph at baseline (2 years prior to onset of illness). (B) Chest radiograph at presentation, demonstrating cardiomegaly and bilateral patchy consolidation in lower lobes. (C) Chest radiograph 8 months after initial presentation showing interval resolution of lung consolidation. Patient underwent drainage of pericardial effusion followed by 6 months of antimicrobial treatment for pericardial actinomycosis. 
of microbiological and histopathological findings, together with clinical response to culture-directed therapy, made the alternative diagnosis of incidental contamination extremely unlikely.

Pericarditis due to anaerobic bacteria remains a rare entity; the true incidence may be underestimated due to a lack of or inadequate anaerobic culture techniques. ${ }^{1}$ The mechanism of occurrence is postulated to arise via spread from a contiguous site of infection (e.g. pleuropulmonary), via haematogenous infection, or direct inoculation resulting from a penetrating injury or surgery.

In our patient, we postulate that pericardial actinomycosis occurred via pleuropulmonary spread, with periodontal disease as a known risk factor for this rare condition. ${ }^{2}$ Pericardial involvement is an uncommon manifestation of actinomycosis, seen in $<2 \%$ of cases. ${ }^{3}$ The condition frequently goes unrecognised due to a low rate of positivity in cultures. ${ }^{2}$ Clinical clues to the identity of the causative organism (e.g. draining sinus tracts, with presence of sulphur granules) are often absent in pericardial actinomycosis, and histologic examination of tissue samples is often necessary to make the diagnosis. ${ }^{2}$

Treatment generally involves at least 4-6 weeks of effective intravenous antimicrobials, followed by $6-12$ months of suppression with oral antibiotics, in view of the high risk of relapse. ${ }^{2}$ While pericardial actinomycosis can evolve into cardiac empyema, presentation as cardiac tamponade is uncommon and has only been reported in isolated case reports. ${ }^{3-5}$ Concomitant recovery of other anaerobic organisms (e.g. Fusobacterium spp.) has also been demonstrated, ${ }^{2,4,5}$ especially with greater usage of molecular techniques.

In conclusion, pericardial actinomycosis presenting as cardiac tamponade is uncommon. Early recognition is crucial given potential mortality, ${ }^{5}$ and complete cure is achievable with appropriate surgical intervention and antimicrobial treatment. In this case, close cooperation and communication among the microbiologist, pathologist, infectious disease physician and surgeon upon suspicion of this rare condition was critical in enabling timely microbiological confirmation of pericardial actinomycosis and institution of appropriate treatment.

\section{REFERENCES}

1. Brook I. Pericarditis caused by anaerobic bacteria. Int $\mathrm{J}$ Antimicrob Agents 2009;33:297-300.

2. Fife TD, Finegold SM, Grennan T. Pericardial actinomycosis: case report and review. Rev Infect Dis 1991;13:120-6.

3. Orloff JJ, Fine MJ, Rihs JD. Acute cardiac tamponade due to cardiac actinomycosis. Chest 1988;93:661-3.

4. Nishizawa S, Anan K, Tobino K, et al. "Pulmonary Actinomycosis attributable to Actinomyces meyeri presenting as cardiac tamponade: a case report”. Multidiscip Respir Med 2018;13:19.

5. Reisinger A, Matzkies LM, Eller P, et al. Pericardial empyema due to Actinomyces israelii, Aggregatibacter actinomycetemcomitans, and Fusobacterium nucleatum. Pol Arch Intern Med 2019;714-5.

Liang En Wee ${ }^{1} M R C P$, Ai Ling Tan ${ }^{2}{ }_{F R C P A}$, Siang Hui Lai ${ }^{3}{ }_{\text {FRCPath }}$, Karrie Kwan-Ki Ko ${ }^{2}{ }_{\text {FRCPath, }}$ Ing Xiang $\underline{\text { SoO }}{ }^{4}$ FRCSEd, Jenny Guek-Hong Low ${ }^{1}$ MRCP

\footnotetext{
${ }^{1}$ Department of Infectious Diseases, Singapore General Hospital, Singapore

${ }^{2}$ Department of Microbiology, Singapore General Hospital, Singapore

${ }^{3}$ Department of Anatomical Pathology, Singapore General Hospital, Singapore

${ }^{4}$ Department of Cardiothoracic Surgery, National Heart Centre Singapore, Singapore
}

Correspondence: Dr Liang En Wee, Department of Infectious Diseases, Singapore General Hospital, Outram Road, Singapore 169608. Email: ian.wee.1.e@singhealth.com.sg 